\title{
Microglial heterogeneity: distinct cell types or differential functional adaptation?
}

\author{
Savannah D. Benusa ${ }^{1,2}$, Nicholas M. George ${ }^{3}$, Jeffrey L. Dupree ${ }^{1,2}$ \\ 'Department of Anatomy and Neurobiology, Virginia Commonwealth University, Richmond, VA 23298, USA. \\ ${ }^{2}$ Research Service, HH McGuire Veterans Affairs Medical Center, Richmond, VA 23249, USA. \\ ${ }^{3}$ Neuroscience Program, University of Colorado Anschutz Medical Campus, Aurora, CO 80045, USA.
}

Correspondence to: Dr. Jeffrey L. Dupree, Department of Anatomy and Neurobiology, Virginia Commonwealth University, 1101 East Marshall Street, Richmond, VA 23298, USA. E-mail: jeffrey.dupree@vcuhealth.org

How to cite this article: Benusa SD, George NM, Dupree JL. Microglial heterogeneity: distinct cell types or differential functional adaptation? Neuroimmuno/ Neuroinflammation 2020;7:248-63. http://dx.doi.org/10.20517/2347-8659.2020.03

Received: 7 Jan 2020 First Decision: 26 Feb 2020 Revised: 15 Mar 2020 Accepted: 31 Mar 2020 Available online: 24 Jun 2020

Science Editor: Jeffrey Bajramovic Copy Editor: Jing-Wen Zhang Production Editor: Tian Zhang

\begin{abstract}
Microglia were first characterized by del Rio Hortega about 100 years ago but our understanding of these cells has only gained traction in the last 20 years. We now recognize that microglia are involved in a plethora of activities including circuitry refinement, neuronal and glial trophic support, cell number modulation, angiogenesis and immune surveillance. Specific to immune surveillance, microglia detect threats which then drive their transformation from ramified to amoeboid cells. This morphological transition is accompanied by changes in cytokine and chemokine expression, which are far less conserved than morphology. To simplify discussion of these expression changes, nomenclature ascribed to states of macrophage activation, known as Macrophage 1 ("M1"; classic) and Macrophage 2 ("M2"; alternative), have been assigned to microglia. However, such a classification for microglia is an oversimplification that fails to accurately represent the array of cellular phenotypes. Additionally, multiple subclasses of microglia have now been described that do not belong to the "M1/M2" classification. Here, we provide a brief review outlining the prominent subclasses of microglia that have been described recently. Additionally, we present novel NanoString data demonstrating distinct microglial phenotypes from three commonly used central nervous system inflammation murine models to study microglial response and conclude with an introduction of recent RNA sequencing studies. In turn, this may not only facilitate a more appropriate naming scheme for these enigmatic cells, but more importantly, provide a framework for generating microglial expression "fingerprints" that may assist in the development of novel therapies by targeting disease-specific microglial subtypes.
\end{abstract}

Keywords: Microglia, neuroinflammation, single cell RNA-seq, NanoString 


\section{INTRODUCTION}

Microglia, the resident immune cells of the central nervous system (CNS), were first characterized 100 years ago by Pio del Rio Hortega (reviewed in ${ }^{[1]}$ ) but our understanding of their function remains incomplete. The best-known function of microglia is CNS surveillance whereby cell debris are scavenged during periods of pathology to maintain and re-establish a healthy homeostatic environment. However, this limited view of microglia function has evolved to include a list of other potential functions designed to establish, maintain, and when necessary, re-establish CNS homeostasis following both pathologic events and in the developing and mature healthy brain. This new appreciation for the plethora of microglial functions in both health and disease has resulted in a renewed interest in these enigmatic and mercurial cells.

At present, it is unclear how microglia are capable of mediating a wide range of activities that, in some cases, are seemingly in contrast to each other. For example, during development, microglia regulate neuronal numbers by both driving cell death ${ }^{[2-7]}$ and promoting proliferation and survival ${ }^{[8-11]}$. This dichotomy of neurogenesis regulation is not limited to the developing brain since microglia both enhance ${ }^{[12]}$ and deplete ${ }^{[13]}$ the number of neural progenitor cells in the adult brain. Similarly, microglia regulate synapse numbers by both stripping/pruning ${ }^{[14-18]}$ and stabilizing ${ }^{[19,20]}$ dendritic spines and inhibitory synapses both in development and adulthood by potentially distinct mechanisms ${ }^{[21-24]}$. Furthermore, microglial regulation of cell populations is not limited to neurons as similar observations have also been reported for oligodendrocytes and astrocytes. Additionally, under pathologic conditions in the adult brain, microglia influence astrocytic phenotypes by ranging from neuroprotective to neurotoxic ${ }^{[25,26]}$ and have been implicated in angiogenesis including regulation of the structure and function of the neurovasculature ${ }^{[27,28]}$. Taken together, it is becoming apparent that microglia oversee a vast array of events in the developing, healthy and diseased CNS although how such a single cell type can manage such a multitude of functions remains to be determined. Strong evidence is now emerging that microglia present as distinct subclasses but it remains to be determined if these subclasses represent intrinsically distinct cell populations, or if intrinsically similar cells are driven into functional heterogeneity dictated by changes in environmental cues provided by a highly dynamic $\mathrm{CNS}^{[29]}$.

In addition to providing a brief review of several parameters and subclasses that define microglial heterogeneity, we also present novel RNA expression profile data that are consistent with the development of distinct microglial phenotypes as a consequence of distinct inflammatory environments. As presented in more detail below, we isolated cortical microglia from mice in three commonly used models to study various aspects of multiple sclerosis - cuprizone, lipopolysaccharide (LPS) and experimental autoimmune encephalomyelitis (EAE). Orally administered cuprizone results in CNS demyelination secondary to oligodendrocyte death. Intraperitoneal injection of the endotoxin LPS mediates a peripheral immune response that results in widespread CNS neuroinflammation. Similarly, EAE is induced by a peripheral injection of a bacterial exotoxin that is accompanied by Complete Freund's Adjuvant and a myelin antigen resulting in breakdown of the blood brain barrier. Although microglia from all three models presented proinflammatory profiles, the microglia from each expressed a unique set of factors suggesting environmentalspecific responses. Although these observations are consistent with environmental cues driving heterogeneity, it remains possible, and perhaps likely, that microglia also represent intrinsically distinct populations.

\section{Microglial heterogeneity}

Currently, a prevailing thought is that microglia, which derive from the embryonic yolk sac, develop initially as a single-cell type lineage ${ }^{[30]}$ and subsequently, into a heterogeneous population in the adult brain as a result of local environmental cues that define their differentiation and functional specificity ${ }^{[31-35]}$. For example, in the injured adult brain, neurons can express or secrete "find me" signals such as fractalkine/ 
C-X3-C motif chemokine receptor $1(\mathrm{Cx} 3 \mathrm{Cr} 1)^{[36]}$ and "eat me" signals such as calreticulin/low-density lipoprotein receptor related protein ${ }^{[37]}$. These signals, which are not present in the healthy CNS, cue microglia to assume a phagocytic phenotype and to "find" and "eat" the compromised cells. Therefore, environmental cues have the capacity to drive the transformation of microglia from a surveying to a phagocytic phenotype. It remains unclear if all microglia in the neighborhood of the "find me" and "eat me" signals respond in the same manner, or if intrinsic heterogeneity results in the response from select subclasses of the microglial population.

\section{Heterogeneity between brain regions}

Some of the earliest evidence of heterogeneity within the microglial population was presented by Lawson et al. ${ }^{[38]}$, who reported brain-region specific densities of cells with higher densities in the hippocampus and thalamus, and a lower density in the cerebellum. Although no functional differences were established, such density differences are consistent with local environmental cues regulating the microglial population. In line with this idea, De Biase et al. ${ }^{[39]}$ reported that regional differences are tightly and specifically regulated since closely apposed nuclei within the basal ganglia present with dramatically different microglial densities ${ }^{[40]}$ while other cell types in the same basal ganglia nuclei present with uniform densities, indicating that differential cell densities are not dictated by the spatial constraints of the tissue. Precisely how these region-specific differences are regulated remains to be fully explained although region-specific self-renewal rates have been presented ${ }^{[41]}$ and it is possible that region-specific cues regulate proliferation and, ultimately, cell density ${ }^{[42]}$. Moreover, factors that regulate microglia numbers in the embryonic brain vs. the adult brain may also differ ${ }^{[43,44]}$, which would be consistent with local cues defining both distribution and heterogeneity within the microglial population. This concept was supported by Grabert et al. ${ }^{[45]}$, who demonstrated that microglia have regionally distinct transcription profiles.

\section{Heterogeneity between sexes}

Variations in cell density and transcription profiles are not limited to regional differences as similar distinctions have also been reported between microglia from male and female mice. Male mice present with more microglia in the cortex, hippocampus, dentate gyrus, and amygdala in early postnatal brains. As the mice mature, these densities flip with female mice presenting with a greater cell density in these regions ${ }^{[46]}$. Although there is no direct evidence that sex-dependent differences in cell density are responsible for functional differences, studies have shown that male and female microglia are functionally distinct and respond differently to noxious stimuli ${ }^{[47,48]}$. For example, Nelson et al. ${ }^{[49]}$ and Yanguas-Casás et al. ${ }^{[40]}$ showed that female microglia have a greater phagocytic capacity but male microglia have greater migratory activity under both basal and interferon $\gamma$-induced inflammatory conditions. Guneykaya et al ${ }^{[50]}$ then reported that male microglia display a higher antigen-presenting capacity as compared to female cells. Interestingly, microglia may also play a role in sex determination since the inhibition of microglial activity in male rodent neonates, at an age critical for sex determination, resulted in the reduction of masculine dendritic spine density and altered copulatory behavior in adults ${ }^{[51]}$. A potential caveat to this work, however, was that microglial activity was inhibited by minocycline, which is a broad spectrum antibiotic that is known to target both $\mathrm{T}$ cells and astrocytes ${ }^{[52,53]}$.

\section{Intrinsically defined heterogeneity?}

The mechanisms responsible for these sex differences are not known and transcriptomic studies comparing male and female microglia reveal expression differences in both the healthy and perturbed states ${ }^{[48,50,54,55]}$. Whether microglia are intrinsically distinct between males and females, or if the local sex-specific environment differentially regulate male and female cells remains to be determined. Microglia from male $v s$. female mice express different sex hormone receptors ${ }^{[56-58]}$ however, and present with sex-specific outcomes when exposed to these hormones ${ }^{[49,51,59-61]}$. Independent of sex, microglia have also been shown to express different levels of markers in the adult, unchallenged brain ${ }^{[62]}$. Bertolotto et al ${ }^{[62]}$ showed that microglia 
express varying levels of keratin sulfate proteoglycan (KSPG) and these microglia are not uniformly distributed throughout the brain, with high concentrations in the hippocampus, brainstem and olfactory bulb while few were found in the cerebellum and cortex. The presence of these $\mathrm{KSPG}^{+}$microglia was independent of development though, since they were found in the same regions of both the neonatal and adult CNS. Moreover, microglia have also been shown to respond differently to the same stimuli ${ }^{[63,64]}$. Although consistent with the involvement of environmental cues in defining subclasses, these findings are also consistent with microglia being intrinsically distinct and independent of environmental influences.

\section{Heterogenic microglial morphology}

Amoeboid vs. ramified microglia

Perhaps the most recognized heterogenic aspect of microglia is their morphology. Two main classes have been identified - amoeboid-like, with few processes; and ramified, with numerous thin, highly-branched processes. Following initial colonization of the embryonic CNS, the majority of microglia present with an amoeboid-like morphology ${ }^{[65,66]}$. With CNS maturation, microglia transform their shape with brain region specificity. In the steady state CNS, amoeboid-like microglia are more abundant in perivascular white matter regions. In contrast, the extent of ramified microglia varies among regions with cerebellar microglia presenting with a less ramified morphology compared to microglia in the cortex ${ }^{[38,67,68]}$. Interestingly, Hanamsagar et al. ${ }^{[69]}$ reported heterogeneity with regard to sex as microglia from male rodents presented with a greater and more complex process of arborization, and exhibited a greater change in process morphology following LPS perturbation as compared to their female counterparts. With age, and as the local environment changes, amoeboid-like microglia become more ramified while ramified microglia transition into amoeboid-like microglia, exhibiting greater phagocytic activity and releasing pro-inflammatory cytokines following pathologic insult ${ }^{[70,71]}$. Although the use of the amoeboid/ramified classification provides a simple approach for discussion, microglial morphologies present a spectrum of shapes and a two-class scheme is insufficient to accurately describe microglial morphologic differences.

\section{Dark microglia}

Recently, a new class of microglia was identified based on morphology. These microglia are "dark" based on their electron dense cytoplasm and are observed in non-homeostatic conditions ${ }^{[72]}$. Dark microglia exhibit signs of oxidative stress including condensed cytoplasm and nucleoplasm (consistent with their name), disrupted mitochondria and dilated endoplasmic reticulum, and are frequently observed extending processes toward synaptic clefts consistent with a role in pathologic synaptic pruning. Although their precise role remains to be fully determined, Bisht and colleagues ${ }^{[72]}$ have proposed that these cells constitute a subclass of hyperactive microglia with dysregulated interactions with synapses. If correct, these cells may play a critical role in the progression of a plethora of neurodegenerative diseases with known synaptic $\operatorname{loss}^{[73]}$ including Alzheimer's Disease ${ }^{[74,75]}$ and multiple sclerosis ${ }^{[76,77]}$.

\section{Axon initial segment-associated microglia}

Baalman et al ${ }^{[78]}$ have also presented evidence of a subset of microglia known as axon initial segmentassociated (AXIS) microglia ${ }^{[78]}$. AXIS microglia, which comprise $~ 8 \%$ of cortical microglia, establish an intimate association with the neuronal cell body and the proximal axon, in contrast to "satellite" microglia that associate with the neuronal cell body and proximal dendrites instead ${ }^{[68]}$. AXIS microglia, which are initially observed at postnatal day 9 and persist through adulthood, contact both inhibitory and excitatory neurons but present with a significant preference for axon initial segments (AISs) of excitatory pyramidal neurons of layer $\mathrm{V}$ of the cortex ${ }^{[78]}$. The function of AXIS microglia is not known but they may provide trophic support for the neuron and the AIS.

Upon activation following a controlled cortical impact (CCI)-induced traumatic brain injury, the association between CNS microglia and the AIS is lost, consistent with the regulation of microglial 

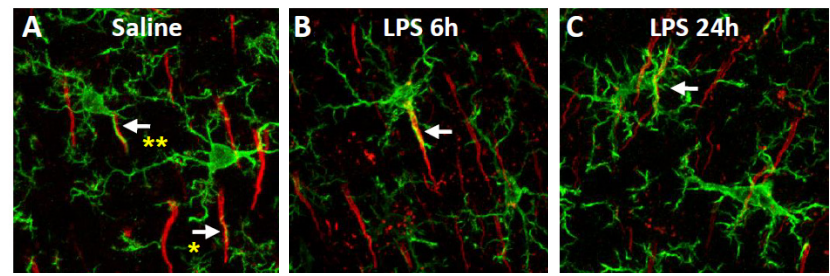

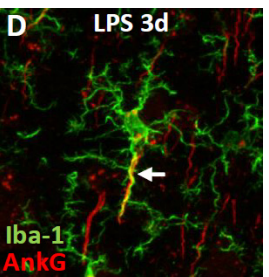

$\mathbf{G}$

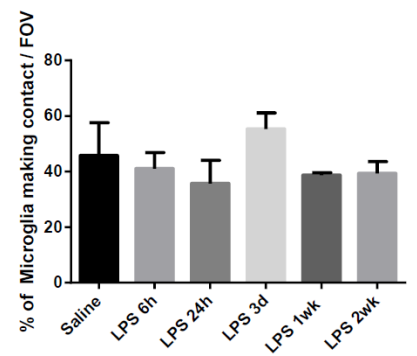

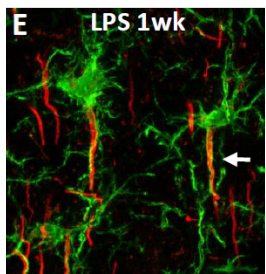

H
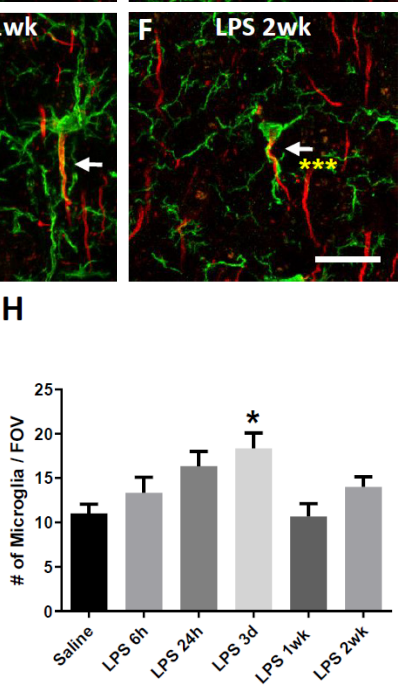

Figure 1. Frequency of microglial-AIS contact is not altered in LPS-induced neuroinflammation. Female c57black6 mice were given a single intraperitoneal injection of LPS $(5 \mathrm{mg} / \mathrm{kg}$ ) or vehicle $(0.9 \%$ saline, $10 \mathrm{~mL} / \mathrm{kg})$. Confocal z-stacks spanning an optical thickness of $25 \mu \mathrm{m}$, using a pinhole of 1 Airy disc unit and Nyquist sampling (optical slice thickness, $0.48 \mu \mathrm{m}$ ), were collected from neocortical layer $\checkmark$ for each of six sections (spanning $1.1 \mathrm{~mm}$ anterior to the bregma to $2.5 \mathrm{~mm}$ posterior to the bregma) per mouse, resulting in 12 images per animal ( $n=4-6$ animals per treatment group). Microglial-AIS contact was quantitatively analyzed at $6 \mathrm{~h}-2$ weeks post-injection in a blind manner using Volocity ${ }^{\mathrm{TM}}$ 3D Image Analysis Software, allowing each confocal z-stack to be observed in three dimensions. The number of microglia, AISs, and contact points in each double immunolabeled z-stack was counted manually. Contact points along the six edges of the z-stacks were excluded from analysis. A-F: double immunolabeling of Iba-1 and AnkG revealed that microglia (Iba-1, green) contact AISs (AnkG, red) (white arrows) in the cortex of saline- and LPS-injected mice; G: the mean \pm SEM of microglia making AIS contact per FOV in saline- and LPS-treated mice as a percent of saline controls. Quantitation of confocal z-stacks revealed that $\sim 45 \%$ of microglia contact AISs in the cortex of saline-injected control mice. Contact was defined by co-localization of Iba- 1 and AnkG and included process touching ( $A$, yellow single asterisk), process alignment ( $A$, yellow double asterisk), and process wrapping ( $F$, yellow triple asterisk) as defined by 3D analysis. No change in the percent of microglia making contact was observed throughout the course of LPS-induced neuroinflammation; $\mathrm{H}$ : the mean $\pm \mathrm{SEM}$ of the number of Microglia/FOV. A significant increase in the number of microglia was observed at 3 days post-LPS injection. Data were statistically compared by one-way ANOVA where mean differences were significant as assessed using Tukey's post hoc analysis. An asterisk indicates a significant difference $(P<0.05)$ from saline. Scale bar $=20 \mu \mathrm{m}$. LPS: lipopolysaccharide; FOV: field of view; AISs: axon initial segments

function and response by the local environment. Interestingly, our laboratory has also reported contact between microglia and the AIS ${ }^{[79,80]}$. Using three dimensional (3D) analysis encompassing multiple types of contact, which was defined by colocalization of ionized calcium binding adaptor molecule 1 (Iba-1) and AnkyrinG, termed (1) process touching, (2) process wrapping, or (3) process alignment [Figure 1], we found that $\sim 45 \%$ of microglia in cortical layer V contact AISs. In contrast to the loss of contact observed following CCI injury, we observed a maintained [Figure 1], and even increased ${ }^{[79]}$, association between the microglia and the AIS following inflammatory insults of LPS injection and EAE induction, respectively. The difference in AXIS microglial responses to insult is intriguing and requires further study to fully elucidate microglial response to pathology.

Herein, we have reviewed several subclasses of microglia that have been defined based on morphology; however, it is unclear if these subclasses are truly distinct, or if they are merely the consequence of artificial classifications based on techniques used for identification, and loose criteria for defining subtypes (reviewed ${ }^{[29]}$ ). If the latter is the case, then there is likely considerable overlap among these 
subclasses. For example, we reported that under certain pathologic conditions, microglia exhibit both an increased association with, and disruption of the $\mathrm{AIS}^{[79]}$. It is possible that these microglia are no longer providing trophic support for the AIS, a suggested function of AXIS microglia by Baalman et al. ${ }^{[78]}$ during homeostatic conditions, but are actively attacking the AIS, perhaps through the release of reactive ions ${ }^{[81]}$. If so, then could these microglia, which we characterized using immunocytochemical approaches, in fact, be "dark microglia", which are identified by electron microscopy? Studies to address this question are currently underway. In addition, are the AXIS microglia, as described by Baalman et al. ${ }^{[78]}$, the same subclass as the microglia we have described making AIS contact? Based on work from Baalman et al. ${ }^{[78]}$, it is likely that the AXIS microglia are supporting the neuron and the AIS; but based on our observations, the microglia may be mediating AIS disruption instead. Answering these questions is essential for accurately classifying microglia but more importantly, it would help to fully understand the role that these mercurial cells play under different conditions.

\section{Heterogenic microglial transcriptomes}

Surveying vs. reactive ("M1/M2") microglia

In an effort to more conclusively characterize microglia and to elucidate their functions, morphologic characterizations have been complemented by molecular classification studies. Initial attempts were based on presumed states of activity based on limited expression profiles. Simply, microglia were classified as either "activated" or "resting" but both terms are misleading. Microglia are never "resting" as we now recognize that they are constantly extending and retracting their processes to survey their surroundings ${ }^{[82,83]}$. As a result, the term "surveying", which more accurately represents the state of activity of microglia, even under homeostatic conditions, is now used in place of "resting". Similarly, a more appropriate term for "activated" is "reactive". "Activated" implies a lack of activity until microglia are stimulated. Microglia are constantly active however, and upon detection of changes in the environment, become "reactive".

Reactive microglia have been further divided into "M1" and "M2" states, referring to the classical (proinflammatory) and alternative (resolving/anti-inflammatory) phenotypes based on expression profiles. The "M1" and "M2" nomenclature is a naming scheme originally derived from the T cell literature and applied to macrophages based on their state of activation in vitro following exposure to either the $\mathrm{T}$ helper type 1 (Th1) cytokine interferon gamma (IFN- $\gamma$ ) for the "M1" phenotype, or the T helper type 2 (Th2) cytokine interleukin 4 (IL-4) for the "M2" phenotype ${ }^{[84]}$. Based on speculation of similar functions between macrophages and microglia, the "M1" and "M2" classification was then applied to microglia. The advantage of the "M1/M2" classification is that it provides a simplified nomenclature to distinguish between microglia in functionally distinct states. However, these distinct states are frequently identified by a small subset of surface markers, which limits resolution required for appreciating heterogeneity that is defined by the entire transcriptome. Moreover, this naming scheme is based on assumptions that cannot be confirmed under close scrutiny. At best, the "M1/M2" classification is inadequate for accurate description of the complex functions of these cells (reviewed by ${ }^{[85,86]}$ ). With the recognized inadequacies of the "M1/ M2" nomenclature, it has been postulated that a continuum of activity states exists between the polarized extremes, resulting in studies presenting "M1" subtypes to better represent the heterogenic nature of these reactive cells ${ }^{[87,88]}$. Recent studies however, have shown that factors assigned to either the "M1" or "M2" phenotype are promiscuous yielding low fidelity to their assigned reactive state ${ }^{[88-90]}$. Thus, the complexity of microglia function is undermined by the overly simplistic and polarized naming scheme of "M1/M2".

\section{Disease-associated microglia}

Another subclass of reactive microglia that is specific to non-homeostatic conditions is known as Disease-Associated Microglia (DAM). First identified in Alzheimer's disease and amyotrophic lateral sclerosis models ${ }^{[91]}$, DAM or microglia with DAM-like phenotypes have now been described in tauopathy models $^{[92,93]}$ multiple sclerosis ${ }^{[94]}$ and aging ${ }^{[91,95]}$. DAM express typical microglia markers including Iba-1, 
cystain 3 and hexosaminidase subunit beta. DAM can downregulate homeostatic genes including P2ry12, $\mathrm{C} \times 3 \mathrm{Cr} 1$, and transmembrane protein 119 (Tmem119), and upregulate genes in either a triggering receptor expressed on myeloid cells 2 (Trem2) dependent (Axl, C-type lectin domain containing 7A, secreted phosphoprotein 1) or independent (Apolipoprotein E, TYRO protein tyrosine kinase-binding protein) manner ${ }^{[91]}$. Interestingly, another related class of microglia, which present with a similar expression profile as $\mathrm{DAM}^{[94]}$, was recently described and named microglial degenerative phenotype $(\mathrm{MGnD})$. It remains to be determined if MGnD and DAM represent the same subclass of cells.

Although unique to non-homeostatic conditions, the function of DAM is not known. It has been hypothesized that these cells respond to a CNS stress signaling system that is akin to the peripheral immune system's pathogen- and damage-associated stress signals (PAMPs and DAMPs) ${ }^{[96]}$. In this scenario, danger signals are recognized by microglia and trigger the transition of surveying microglia into DAM. This hypothesis is consistent with DAM accumulation in Alzheimer's Disease plaques and regions of demyelination ${ }^{[1,94,97,98]}$. If correct, DAM would be a key component of an intrinsic mechanism designed to combat disease processes and could provide a promising target for therapeutic manipulation against neurodegenerative disease by further enhancing the DAM response.

\section{Heterogenic expression in inflammatory microglia}

Following injury or disease, reactive microglia are rapidly recruited to sites of damage where they phagocytose debris and dying cells, consistent with the described functions of DAM. Likewise, AXIS microglia may also be recruited to sites of damage following injury or disease ${ }^{[80,81]}$. However, unlike DAM, the expression profile of AXIS microglia has not been characterized. Instead, AXIS microglia have been characterized based on their physical interactions with the axonal domain of the AIS. Both surveying and reactive microglia make contact with AISs and this is increased or decreased based on the disease context $^{[78,79]}$. Whether these cells provide trophic support at the AIS or drive pathogenesis remains unclear though. Reactive microglia also exhibit extensive changes in expression of their inflammatory profile ${ }^{[99]}$. While some of these secreted factors may provide neurotrophic functions, pro-inflammatory factors can also exhibit deleterious effects ${ }^{[100,101]}$. For example, pro-inflammatory microglia ("M1") upregulate enzymes that produce reactive oxygen species (ROS $)^{[100]}$. Activation of microglial nicotinamide adenine dinucleotide phosphate (NADPH) oxidase $(\mathrm{NOX} 2)$ results in the extracellular production of $\mathrm{ROS}^{[102]}$. ROS then alter the function of calcium-permeable ion channels ${ }^{[103-105]}$ and consequently, alters intracellular calcium levels ${ }^{[105,106]}$, which have been implicated in AIS disruption ${ }^{[81,107-110]}$.

In addition to regulating neuronal function through secreted factors, microglia also regulate neurons through physical contact ${ }^{[82,111-115]}$. In the developing and adult brain, microglia contact pre- and postsynaptic neuronal elements in an activity-dependent manner, and synapses that are contacted more frequently are subsequently removed ${ }^{[17,115,116]}$. In pathological conditions, microglia participate in synaptic stripping altering the neuronal excitatory/inhibitory balance ${ }^{[16]}$. Microglia also preferentially contact cell bodies and axons of highly active neurons to decrease neuronal activity and prevent excitotoxic cell death ${ }^{[13,114]}$. These studies underscore the importance of microglial contact in the regulation of neural signaling.

Recently, we analyzed CNS pathology in three models of neuroinflammation. In all three models, microglia presented with reactive phenotypes and these cells maintained, or even increased, contact with the AIS. However, in two of the models, the AISs were disrupted and in one, the AISs were preserved. Since AIS integrity temporally correlated with the presence of reactive microglia and contact was at least maintained in all three models, we proposed that differential AIS integrity was consequential to the heterogeneity among the reactive microglia from all three models.

For our studies, we exploited the immune-mediated inflammatory models of EAE ${ }^{[79]}$, LPS $^{[80]}$ and the demyelinating model of cuprizone ${ }^{[79]}$. The EAE model is induced through subcutaneous injection of 
myelin peptide (myelin oligodendrocyte glycoprotein peptide 35-55) accompanied by pertussis toxin and an adjuvant to ignite an inflammatory response $\mathrm{e}^{[117-119]}$, which transfers to the brain and results in chronic neuroinflammation persisting for months. While neuroinflammation is present after induction and throughout the EAE course, clinical symptoms do not begin to appear until $\sim 15$ days post-induction ${ }^{[79]}$. Mice exhibit a range of clinical symptoms from limp tail and loss of righting reflex (EAE 1 \& 2, respectively) to single- or double- hind limb paralysis (EAE $3 \& 4$, respectively $)^{[79]}$. AIS pathology begins to appear at an early timepoint after clinical onset ( 18 days post-induction), but only in mice that display more severe clinical symptoms (EAE Early $3 \& 4$ ). Increased AIS pathology is observed with disease severity and progression (EAE Late $1 \& 2,3 \& 4, \sim 25$ days post-induction) ${ }^{[79]}$. In contrast, the LPS model is an acute neuroinflammatory model induced by a single peripheral injection of LPS $^{[120,121]}$. This results in widespread peripheral inflammation that rapidly transfers to the brain $(\sim 3 \mathrm{~h})$, but the neuroinflammation is resolved by 2 weeks post-injection. In the LPS model, AIS pathology was present from as early as $24 \mathrm{~h}$ post-injection and persisted until 1 week post-injection, coincident with the initiation and resolution of the acute neuroinflammatory environment ${ }^{[80]}$. In contrast to the immune-mediated neuroinflammatory models, the cuprizone model is a demyelinating model ${ }^{[79]}$ where a copper-chelating toxin, cuprizone, is administered through chow resulting in oligodendrocyte cell death and, consequently, loss of myelin ${ }^{[119]}$. Demyelination is detectable 1-2 weeks after cuprizone treatment with peak demyelination occurring by 5-6 weeks of exposure $^{[122-124]}$. The cuprizone model yields substantial cell death and demyelination resulting in microglial recruitment and neuroinflammation but no AIS pathology was observed ${ }^{[79]}$.

We utilized these three models to further investigate microglial heterogeneity. AIS disruption only occurred in the LPS and EAE models, while microglial-AIS contact was abundant in all three models. Thus, while microglial reactivity and contact increased prior to and was coincident with disruption in EAE, contact alone did not disrupt AIS integrity ${ }^{[79,80]}$. Therefore, we analyzed the inflammatory expression profiles of cortical microglia across all three models to assess how microglial reactivity differentially influences neuronal integrity. Our goal was to assess microglia expression profiles early in the disease process to identify inflammatory changes that drive disease progression and are not consequential of disease progression. Thus, cortical microglia were isolated from mice induced with EAE, Cuprizone, or LPS at time points where neuronal pathology is detectable but had not peaked (EAE Early $3 \& 4^{[79]}$, 3 week Cuprizone ${ }^{[123]}$, LPS $24 \mathrm{~h})^{[80]}$. Briefly, total RNA, collected from cluster of differentiation (CD) $11 \mathrm{~b}^{+}$cells isolated from the cortex of c57black6 female mice, was submitted for NanoString mRNA expression analysis. (Further details on model generation, cell isolation and NanoString analyses are provided in Supplementary Materials $\left.{ }^{[79,80,120-123,125-133]}\right)$. Cells were collected at time points in each model that corresponded to the early presence of neuronal/myelin pathology, but prior to peak disease course in an effort to understand the inflammatory profiles that drive pathogenesis ${ }^{[79,80]}$.

Microglia with reactive morphologies predominate in the cortex of all three models ${ }^{[79,80]}$ [Figure $\left.2 \mathrm{~A}\right]$, which is consistent with these cells presenting with a pro-inflammatory phenotype. However, based on NanoString expression analysis of 248 inflammation-associated genes, microglia from all three neuroinflammatory models displayed distinct regulation of inflammatory genes [Figure $2 \mathrm{~B}$ ], underscoring the heterogeneity of morphologically similar cells. Of 248 analyzed genes, 95 were significantly upregulated (1.3 fold-change or greater) [Figure $2 \mathrm{C}$ ] and 175 were significantly downregulated (at least 1.3 fold-change) among the three neuroinflammatory models when compared to microglia from naïve mice [Figure 2D]. 27 of $95(28.4 \%)$ upregulated genes [Figure $2 \mathrm{C}$ ] and 50 of $175(28.6 \%)$ downregulated genes [Figure 2D] were similarly changed across all three models but model-specific differences were observed for both categories. Numerous genes [Figure 2C] associated with a pro-inflammatory ("M1") phenotype (such as interferon regulatory factor 1, lymphotoxin beta, C-C chemokine receptor type 7, C-C motif chemokine ligand 7, C-C motif chemokine ligand 17, lymphotoxin Alpha, Il1a, signal transducer and activator of transcription 2, and tumor necrosis factor super family 14) were upregulated uniquely in EAE Early 3 \& 4 and LPS $24 \mathrm{~h}$ 


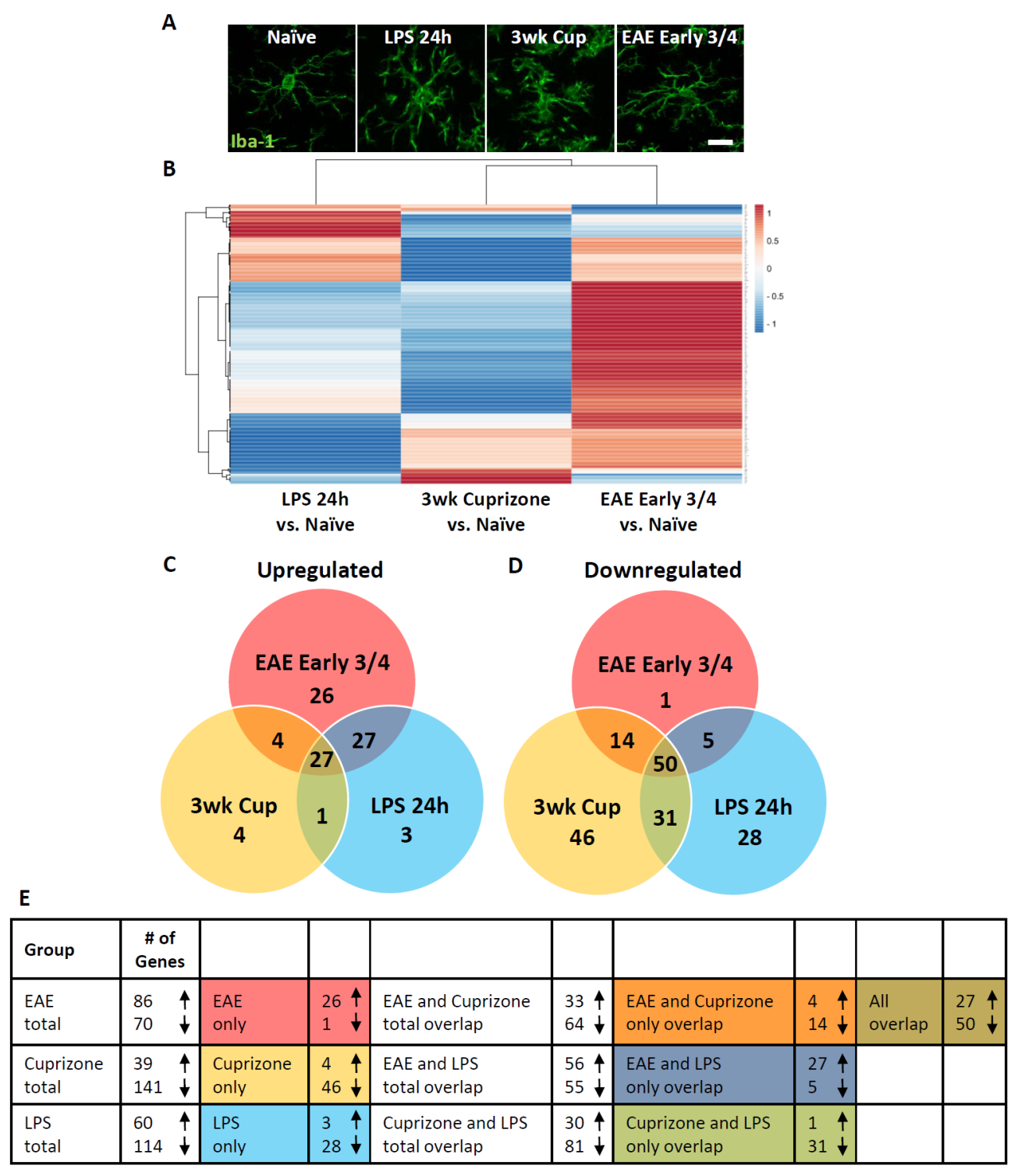

Figure 2. Microglia differentially express inflammation-associated genes in three neuroinflammatory models that demonstrate robust microglial reactivity. A: representative images of surveying microglia from the cortex of naïve mice and reactive microglia from LPS $24 \mathrm{~h}$, 3 week Cuprizone, and EAE Early 3 \& 4 mice; B-D: analysis of NanoString data of 248 differentially expressed inflammation-associated genes in $\mathrm{CD}_{11 \mathrm{~b}^{+}}$cells. Background subtraction was performed using the maximum value across samples of the negative controls and data normalization was performed using the geometric mean expression of six internal reference genes (CLTC, GAPDH, Gusb, Hprt, Pgk7, Tubb5). Reporter probe counts reflecting the numbers of mRNA transcript in the RNA sample were analyzed and quantified using the nSolverTM Analysis Software, and are represented by fold-change compared to naïve cells. Two mice were pooled per sample and three total samples per group were submitted for NanoString analysis. Microglia were isolated by CD11b Miltenyi beads from the cortex of mice induced with EAE, Cuprizone, or LPS at early time points where neuronal pathology was detectable but had not peaked (EAE Early $3 \& 4^{[79]}, 3$ week Cuprizone ${ }^{[123]}$, LPS 24 h) ${ }^{[80]}$; B: heat map of differentially expressed genes; C: venn diagram representing the number of genes that are significantly upregulated, 1.3 fold-change or greater, in microglia from mice induced with EAE, Cuprizone, or LPS; D: venn diagram representing the number of genes that are significantly downregulated, 1.3 fold-change or greater, in microglia from mice induced with EAE, Cuprizone, or LPS; E: table showing the number of genes that were significantly upregulated (upward arrow) or downregulated (downward arrow) in each experimental group, and the number of altered genes shared among groups. Scale bar $=10 \mu \mathrm{m} . P<0.05$. LPS: lipopolysaccharide; EAE: experimental autoimmune encephalomyelitis

mice, consistent with the involvement of infective and inflammatory response pathways ${ }^{[127]}$. Gene ontology biological processes $(\mathrm{GO}-\mathrm{BP})$ function analysis ${ }^{[125,126]}$ revealed that these genes were involved in functions related to regulation of the pro-inflammatory response as defined by the production of tumor necrosis factor alpha, nitric oxide biosynthetic process, and chemotaxis and chemokine signaling. In contrast, 
microglia from 3 week cuprizone treated mice had the greatest number of downregulated inflammatory genes, and the top four uniquely upregulated genes associated with phagocytosis and oligodendrocyte generation [guanine nucleotide-binding protein $\mathrm{G}(\mathrm{s})$ subunit, platelet derived growth factor alpha, TYRO protein tyrosine kinase-binding protein (TyroBP), C-C chemokine receptor type]. Platelet derived growth factor alpha is a mitogen that is critical for oligodendrocyte generation ${ }^{[134]}$. TyroBP is a microglial transmembrane signaling polypeptide that forms phagocytosis active zones preparing microglia for phagocytic activity ${ }^{[135]}$. Increased expression of TyroBP in the 3 week treated cuprizone mice, a treatment time point that corresponds to early myelin loss, is consistent with findings from transcriptome microglial analysis from demyelinating regions in other animal studies ${ }^{[136]}$ and human tissue ${ }^{[137]}$. Thus, while microglia from all three models exhibit pro-inflammatory ("M1"), reactive expression profiles, microglia maintained a unique "fingerprint" for each model and these differences correspond with the integrity of the AIS, suggesting that subtle changes in microglial phenotype may mediate either stability or disruption of closely apposed neurons. It is still possible though that microglial phenotypes do not directly influence AIS integrity. The direct association of microglia with the AIS suggests however, that this neuronal domain may be particularly vulnerable to changes in microglial reactivity. These data support the growing body of literature demonstrating that microglia exhibit a plethora of inflammatory expression profiles within an "M1" phenotype despite having similar morphologies.

\section{Transcriptomic defined subsets of microglia}

Recently, several single cell RNA sequencing studies have begun to more clearly define subsets of microglia in the developing,mature and healthy, and pathologic $\mathrm{CNS}^{[45,138,139]}$. Grabert and colleagues ${ }^{[45]}$ conducted the first genome-wide comparison of RNA expression profiles from microglia isolated from specific brain regions and across the adult life span. Their findings confirmed the presence of core profiles that distinguish microglia from macrophages, underscoring their distinct origins. In addition, they observed three primary RNA profiles that were regionally specific, demonstrating regional heterogeneity within the microglial population. Although regional specific heterogeneity was observed, similarities persisted between the cortex and the striatum, and between the cerebellum and hippocampus. With age, some of these differences dissipated as the profile of hippocampal microglia appeared to converge with the profiles of microglia from the cortex and striatum, while the profile of cerebellar microglia continued to diverge from the other three regions to reveal region specific changes over time. Li et al. ${ }^{[138]}$ reported that the majority of microglia in mature, healthy CNS express similar profiles but significantly greater diversity was seen in postnatal CNS. An interesting finding of Li et al. ${ }^{[138]}$ was the similarity between a postnatal subset of microglia, termed Proliferative-region Associated Microglia (PAM), and DAM, which demonstrates that genes expressed in development are reactivated with aging and pathology. PAM appeared transiently in regions of developing white matter, consistent with a role in phagocytosing the large numbers of oligodendrocytes that die during myelination ${ }^{[140]}$. The authors further state that the complete chemokine and cytokine expression profile of PAM supports additional roles including interacting with both neural and immune cells.

Using fluorescent assisted cell sorting gated by CD11b, CD45 and Cx3Cr1, Hammond et al ${ }^{[139]}$ defined nine unique clusters of microglia in the whole brain based on expression profiles. The percent of cells in each cluster changed across age and condition however. Canonical microglia genes were expressed by most cells but only C1qa, Fcrls and Trem 2 were expressed in all clusters. Interestingly, P2ry12, $\mathrm{Cx} 3 \mathrm{Cr} 1$ and Tmem119, which are frequently used as microglial identifiers ${ }^{[141-143]}$, were either expressed in very low levels, or not at all in some clusters during development. Additionally, a novel subset of microglia, defined by the expression of secreted phosphoprotein 1, similar to PAM described by Li et al ${ }^{[138]}$, insulin like growth factor 1 and immunomodulators from the galectin family and several lysosomal proteins, was observed in the postnatal brain and associated with axonal tracts destined for myelination. Since these microglia express lysosomal markers, it was proposed that these cells clear the way for continued axon outgrowth, ultimately facilitating subsequent myelination. Other interesting findings include the lack of sex differences 
based on cluster comparisons, which is in contrast to previous reports ${ }^{[48,50,54,128]}$. Although sex differences were not observed, significant differences were observed within the aged brain (postnatal day 540) as certain clusters, which were comprised of very few cells in the adult brain (postnatal day 100), revealed a significant increase in the number of cells in the aged brain. Perhaps most interesting is the finding that specific subpopulations of microglia were similarly represented in demyelinating lesions in the mouse and human brains, suggesting that microglial cluster expression profiles may allow identifying disease-specific"fingerprints", and eventually aid in human disease treatment.

\section{CONCLUSION}

Although described 100 years ago, we are only just beginning to put together the various pieces of the microglial puzzle. We now recognize their involvement in establishing and maintaining a homeostatic CNS environment through trophic support and pruning of both neuronal and glial populations, modulating CNS wiring and circuitry, and facilitating axonal organization and outgrowth, myelin formation, and immunosurveillance in the healthy brain. Moreover, we are also beginning to appreciate their critical roles in disease, potentially both as CNS protectors by recognizing and removing infected, dying and dead cells, and also as CNS villains secondary to hyperactivation or dysregulation. We are also beginning to recognize that microglia may present as functionally distinct subclasses, which provides an explanation as to how a single lineage cell type can manifest into a plethora of diverse roles. However, it remains to be determined if distinct subclasses of microglia truly exist, or if microglia exist on a spectrum where they have the capacity to take on a multitude of identities depending on their environment. To address this issue, consistent approaches in cell isolation and analysis should be established and implemented. Additionally, as presented by other authors ${ }^{[86]}$, the generation of a naming scheme that incorporates all aspects (age, brain region, morphology, gene expression, function, etc.) of microglia is essential for effectively moving the field forward. Although much has been learned over the past 20 years, our understanding of microglia remains limited. The immediate future though should be viewed with excitement as we continue to unravel the mysteries of these enigmatic cells.

\section{DECLARATIONS}

\section{Authors' contributions}

Made substantial contributions to experimental conception and design and manuscript preparation: Dupree JL

Made substantial contributions to experimental conception and design, in technical support, mRNA data analysis and interpretation and manuscript preparation: Benusa SD

Made substantial contributions to microglia-AIS contact analysis and interpretation: George NM

\section{Availability of data and materials}

NanoString raw data files are provided in Supplementary Material.

\section{Financial support and sponsorship}

This work was supported by grants from the National Institute of Health ["Microglial neurofascin: a novel mediator of microglial/axon initial segment interactions?" R21NS1016515; (JLD)] and the Veterans Affairs ["Attenuating microglial-dependent axonal pathology in EAE" (No. BX002565 (JLD)]. Microscopy was performed at the VCU Massey Cancer Center Microscopy Core Facility and supported, in part, with funding from NIH-NCI Cancer Center Support Grant P30 CA016059.

\section{Conflicts of interest}

All authors declared that there are no conflicts of interest. 


\section{Ethical approval and consent to participate}

Not applicable.

\section{Consent for publication}

Not applicable.

\section{Copyright}

(c) The Author(s) 2020.

\section{REFERENCES}

1. Tremblay MÈ, Lecours C, Samson L, Sánchez-Zafra V, Sierra A. From the Cajal alumni Achúcarro and Río-Hortega to the rediscovery of never-resting microglia. Front Neuroanat 2015;9:45.

2. Marín-Teva JL, Dusart I, Colin C, Gervais A, van Rooijen N, et al. Microglia promote the death of developing Purkinje cells. Neuron 2004;41:535-47.

3. Swinnen N, Smolders S, Avila A, Notelaers K, Paesen R, et al. Complex invasion pattern of the cerebral cortex bymicroglial cells during development of the mouse embryo. Glia 2013;61:150-63.

4. Wakselman S, Béchade C, Roumier A, Bernard D, Triller A, et al. Developmental neuronal death in hippocampus requires the microglial CD11b integrin and DAP12 immunoreceptor. J Neurosci 2008;28:8138-43.

5. Antony JM, Paquin A, Nutt SL, Kaplan DR, Miller FD. Endogenous microglia regulate development of embryonic cortical precursor cells. J Neurosci Res 2011;89:286-98.

6. Cunningham CL, Martínez-Cerdeño V, Noctor SC. Microglia regulate the number of neural precursor cells in the developing cerebral cortex. J Neurosci 2013;33:4216-33.

7. Tong CK, Vidyadaran S. Role of microglia in embryonic neurogenesis. Exp Biol Med (Maywood) 2016;241:1669-75.

8. Shigemoto-Mogami Y, Hoshikawa K, Goldman JE, Sekino Y, Sato K. Microglia enhance neurogenesis and oligodendrogenesis in the early postnatal subventricular zone. J Neurosci 2014;34:2231-43.

9. Ueno M, Fujita Y, Tanaka T, Nakamura Y, Kikuta J, et al. Layer V cortical neurons require microglial support for survival during postnatal development. Nat Neurosci 2013;16:543-51.

10. Ribeiro Xavier AL, Kress BT, Goldman SA, Lacerda de Menezes JR, Nedergaard M. A distinct population of microglia supports adult neurogenesis in the subventricular zone. J Neurosci 2015;35:11848-61.

11. Oosterhof N, Chang IJ, Karimiani EG, Kuil LE, Jensen DM, et al. Homozygous mutations in CSF1R cause a pediatric-onset leukoencephalopathy and can result in congenital absence of microglia. Am J Hum Genet 2019;104:936-47.

12. Rogers JT, Morganti JM, Bachstetter AD, Hudson CE, Peters MM, et al. CX3CR1 deficiency leads to impairment of hippocampal cognitive function and synaptic plasticity. J Neurosci 2011;31:16241-50.

13. Vukovic J, Colditz MJ, Blackmore DG, Ruitenberg MJ, Bartlett PF. Microglia modulate hippocampal neural precursor activity in response to exercise and aging. J Neurosci 2012;32:6435-43.

14. Šišková Z, Tremblay MÈ. Microglia and synapse: interactions in health and neurodegeneration. Neural Plast 2013;2013:425845.

15. Chen Z, Trapp BD. Microglia and neuroprotection. J Neurochem 2016;136:10-7.

16. Kettenmann H, Kirchhoff F, Verkhratsky A. Microglia: new roles for the synaptic stripper. Neuron 2013;77:10-8.

17. Schafer DP, Lehrman EK, Kautzman AG, Koyama R, Mardinly AR, et al. Microglia sculpt postnatal neural circuits in an activity and complement-dependent manner. Neuron 2012;74:691-705.

18. Hong S, Dissing-Olesen L, Stevens B. New insights on the role of microglia in synaptic pruning in health and disease. Curr Opin Neurobiol 2016;36:128-34.

19. Ziebell JM, Adelson PD, Lifshitz J. Microglia: dismantling and rebuilding circuits after acute neurological injury. Metab Brain Dis 2015;30:393-400.

20. Ikegami A, Haruwaka K, Wake H. Microglia: lifelong modulator of neural circuits. Neuropathology 2019;39:173-80.

21. Paolicelli RC, Bolasco G, Pagani F, Maggi L, Scianni M, et al. Synaptic pruning by microglia is necessary for normal brain development. Science 2011;333:1456-8.

22. Vilalta A, Brown GC. Neurophagy, the phagocytosis of live neurons and synapses by glia, contributes to brain development and disease. FEBS J 2018;285:3566-75.

23. Stevens B, Allen NJ, Vazquez LE, Howell GR, Christopherson KS, et al. The classical complement cascade mediates CNS synapse elimination. Cell 2007;131:1164-78.

24. Parkhurst CN, Yang G, Ninan I, Savas JN, Yates JR, et al. Microglia promote learning-dependent synapse formation through brainderived neurotrophic factor. Cell 2013;155:1596-609.

25. Jha MK, Jo M, Kim JH, Suk K. Microglia-astrocyte crosstalk: an intimate molecular conversation. Neuroscientist 2019;25:227-40.

26. Shinozaki Y, Shibata K, Yoshida K, Shigetomi E, Gachet C, et al. Transformation of astrocytes to a neuroprotective phenotype by microglia via P2Y1 receptor downregulation. Cell Rep 2017;19:1151-64.

27. Singh-Bains MK, Linke V, Austria MDR, Tan AYS, Scotter EL, et al. Altered microglia and neurovasculature in the Alzheimer's disease cerebellum. Neurobiol Dis 2019;132:104589.

28. Zhao X, Eyo UB, Murugan M, Wu LJ. Microglial interactions with the neurovascular system in physiology and pathology. Dev Neurobiol 2018;78:604-17. 
29. Stratoulias V, Venero JL, Tremblay M, Joseph B. Microglial subtypes: diversity within the microglial community. EMBO J 2019;38:e101997.

30. Ginhoux F, Greter M, Leboeuf M, Nandi S, See P, et al. Fate mapping analysis reveals that adult microglia derive from primitive macrophages. Science 2010;330:841-5.

31. McCarthy MM. Location, location, location: microglia are where they live. Neuron 2017;95:233-5.

32. Hickman SE, Kingery ND, Ohsumi TK, Borowsky ML, Wang LC, et al. The microglial sensome revealed by direct RNA sequencing. Nat Neurosci 2013;16:1896-905.

33. Butovsky O, Jedrychowski MP, Moore CS, Cialic R, Lanser AJ, et al. Identification of a unique TGF- $\beta$-dependent molecular and functional signature in microglia. Nat Neurosci 2014;17:131-43.

34. Gosselin D, Link VM, Romanoski CE, Fonseca GJ, Eichenfield DZ, et al. Environment drives selection and function of enhancers controlling tissue-specific macrophage identities. Cell 2014;159:1327-40.

35. Grabert K, Michoel T, Karavolos MH, Clohisey S, Baillie JK, et al. Microglial brain region-dependent diversity and selective regional sensitivities to ageing. Nat Neurosci 2016;19:504-16.

36. Sokolowski JD, Chabanon-Hicks CN, Han CZ, Heffron DS, Mandell JW. Fractalkine is a "find-me" signal released by neurons undergoing ethanol-induced apoptosis. Front Cell Neurosci 2014;8:360.

37. Fricker M, Oliva-Martín MJ, Brown GC. Primary phagocytosis of viable neurons by microglia activated with LPS or A $\beta$ is dependent on calreticulin/LRP phagocytic signalling. J Neuroinflammation 2012;9:196.

38. Lawson LJ, Perry VH, Dri P, Gordon S. Heterogeneity in the distribution and morphology of microglia in the normal adult mouse brain. Neuroscience 1990;39:151-70.

39. De Biase LM, Schuebel KE, Fusfeld ZH, Jair K, Hawes IA, et al. Local cues establish and maintain region-specific phenotypes of basal ganglia microglia. Neuron 2017;95:341-56.e6.

40. Yanguas-Casás N, Crespo-Castrillo A, de Ceballos ML, Chowen JA, Azcoitia I, et al. Sex differences in the phagocytic and migratory activity of microglia and their impairment by palmitic acid. Glia 2018;66:522-37.

41. Tay TL, Savage JC, Hui CW, Bisht K, Tremblay MÈ. Microglia across the lifespan: from origin to function in brain development, plasticity and cognition. J Physiol (Lond) 2017;595:1929-45.

42. Rigato C, Swinnen N, Buckinx R, Couillin I, Mangin JM, et al. Microglia proliferation is controlled by P2X7 receptors in a Pannexin1-independent manner during early embryonic spinal cord invasion. J Neurosci 2012;32:11559-73.

43. Greter M, Lelios I, Pelczar P, Hoeffel G, Price J, et al. Stroma-derived interleukin-34 controls the development and maintenance of langerhans cells and the maintenance of microglia. Immunity 2012;37:1050-60.

44. Wang Y, Szretter KJ, Vermi W, Gilfillan S, Rossini C, et al. IL-34 is a tissue-restricted ligand of CSF1R required for the development of Langerhans cells and microglia. Nat Immunol 2012;13:753-60.

45. Grabert K, Michoel T, Karavolos MH, Clohisey S, Baillie JK, et al. Microglial brain region-dependent diversity and selective regional sensitivities to aging. Nat Neurosci 2016;19:504-16.

46. Schwarz JM, Sholar PW, Bilbo SD. Sex differences in microglial colonization of the developing rat brain. J Neurochem 2012;120:948-63.

47. Posillico CK, Terasaki LS, Bilbo SD, Schwarz JM. Examination of sex and minocycline treatment on acute morphine-induced analgesia and inflammatory gene expression along the pain pathway in Sprague-Dawley rats. Biol Sex Differ 2015;6:33.

48. Villa A, Gelosa P, Castiglioni L, Cimino M, Rizzi N, et al. Sex-specific features of microglia from adult mice. Cell Rep 2018;23:3501-11.

49. Nelson LH, Warden S, Lenz KM. Sex differences in microglial phagocytosis in the neonatal hippocampus. Brain Behav Immun 2017;64:11-22.

50. Guneykaya D, Ivanov A, Hernandez DP, Haage V, Wojtas B, et al. Transcriptional and translational differences of microglia from male and female brains. Cell Rep 2018;24:2773-83.e6.

51. Lenz KM, Nugent BM, Haliyur R, McCarthy MM. Microglia are essential to masculinization of brain and behavior. J Neurosci 2013;33:2761-72.

52. Zhou YQ, Liu DQ, Chen SP, Sun J, Wang XM, et al. Minocycline as a promising therapeutic strategy for chronic pain. Pharmacol Res 2018; 134:305-10.

53. Naderi Y, Panahi Y, Barreto GE, Sahebkar A. Neuroprotective effects of minocycline on focal cerebral ischemia injury: a systematic review. Neural Regen Res 2020;15:773-82.

54. Crain JM, Nikodemova M, Watters JJ. Microglia express distinct M1 and M2 phenotypic markers in the postnatal and adult central nervous system in male and female mice. J Neurosci Res 2013;91:1143-51.

55. Mangold CA, Wronowski B, Du M, Masser DR, Hadad N, et al. Sexually divergent induction of microglial-associated neuroinflammation with hippocampal aging. J Neuroinflammation 2017;14:141.

56. Puy L, MacLusky NJ, Becker L, Karsan N, Trachtenberg J, et al. Immunocytochemical detection of androgen receptor in human temporal cortex characterization and application of polyclonal androgen receptor antibodies in frozen and paraffin-embedded tissues. J Steroid Biochem Mol Biol 1995;55:197-209.

57. Mor G, Nilsen J, Horvath T, Bechmann I, Brown S, et al. Estrogen and microglia: a regulatory system that affects the brain. J Neurobiol 1999;40:484-96.

58. Crain JM, Watters JJ. Estrogen and P2 purinergic receptor systems in microglia: therapeutic targets for neuroprotection. Open Drug Discov J 2010;2:148-67.

59. Vegeto E, Bonincontro C, Pollio G, Sala A, Viappiani S, et al. Estrogen prevents the lipopolysaccharide-induced inflammatory response in microglia. J Neurosci 2001;21:1809-18.

60. Sierra A, Gottfried-Blackmore A, Milner TA, McEwen BS, Bulloch K. Steroid hormone receptor expression and function in microglia Glia 2008;56:659-74.

61. Bollinger JL, Salinas I, Fender E, Sengelaub DR, Wellman CL. Gonadal hormones differentially regulate sex-specific stress effects on 
glia in the medial prefrontal cortex. J Neuroendocrinol 2019;31:e12762.

62. Bertolotto A, Caterson B, Canavese G, Migheli A, Schiffer D. Monoclonal antibodies to keratan sulfate immunolocalize ramified microglia in paraffin and cryostat sections of rat brain. J Histochem Cytochem 1993;41:481-7.

63. Scheffel J, Regen T, Van Rossum D, Seifert S, Ribes S, et al. Toll-like receptor activation reveals developmental reorganization and unmasks responder subsets of microglia. Glia 2012;60:1930-43.

64. Kiyofuji K, Kurauchi Y, Hisatsune A, Seki T, Mishima S, et al. A natural compound macelignan protects midbrain dopaminergic neurons from inflammatory degeneration via microglial arginase-1 expression. Eur J Pharmacol 2015;760:129-35.

65. Silvin A, Ginhoux F. Microglia heterogeneity along a spatio-temporal axis: more questions than answers. Glia 2018;66:2045-57.

66. Marín-Teva JL, Cuadros MA, Martín-Oliva D, Navascués J. Microglia and neuronal cell death. Neuron Glia Biol 2011;7:25-40.

67. Imamura K, Ito M, Suzumura A, Asai J, Takahashi A. Generation and characterization of monoclonal antibodies against rat microglia and ontogenic distribution of positive cells. Lab Invest 1990;63:853-61.

68. Stowell RD, Wong EL, Batchelor HN, Mendes MS, Lamantia CE, et al. Cerebellar microglia are dynamically unique and survey Purkinje neurons in vivo. Dev Neurobiol 2018;78:627-44.

69. Hanamsagar R, Alter MD, Block CS, Sullivan H, Bolton JL, et al. Generation of a microglial developmental index in mice and in humans reveals a sex difference in maturation and immune reactivity. Glia 2017;65:1504-20.

70. Lynch MA. The multifaceted profile of activated microglia. Mol Neurobiol 2009;40:139-56.

71. Diestel A, Troeller S, Billecke N, Sauer IM, Berger F, et al. Mechanisms of hypothermia-induced cell protection mediated by microglial cells in vitro. Eur J Neurosci 2010;31:779-87.

72. Bisht K, Sharma KP, Lecours C, Sánchez MG, El Hajj H, et al. Dark microglia: a new phenotype predominantly associated with pathological states. Glia 2016;64:826-39.

73. Cardozo PL, de Lima IBQ, Maciel EMA, Silva NC, Dobransky T, et al. Synaptic elimination in neurological disorders. Curr Neuropharmacol 2019;17:1071-95.

74. Rajendran L, Paolicelli RC. Microglia-mediated synapse loss in Alzheimer’s disease. J Neurosci 2018;38:2911-9.

75. Jackson J, Jambrina E, Li J, Marston H, Menzies F, et al. Targeting the synapse in Alzheimer's disease. Front Neurosci 2019;13:735.

76. Stadelmann C, Albert M, Wegner C, Brück W. Cortical pathology in multiple sclerosis. Curr Opin Neurol 2008;21:229-34.

77. Campbell G, Licht-Mayer S, Mahad D. Targeting mitochondria to protect axons in progressive MS. Neurosci Lett 2019;710:134258.

78. Baalman K, Marin MA, Ho TS, Godoy M, Cherian L, et al. Axon initial segment-associated microglia. J Neurosci 2015;35:2283-92.

79. Clark KC, Josephson A, Benusa SD, Hartley RK, Baer M, et al. Compromised axon initial segment integrity in EAE is preceded by microglial reactivity and contact. Glia 2016;64:1190-209.

80. Benusa SD, George NM, Sword BA, DeVries GH, Dupree JL. Acute neuroinflammation induces AIS structural plasticity in a NOX2dependent manner. J Neuroinflammation 2017;14:116.

81. Clark K, Sword BA, Dupree JL. Oxidative stress induces disruption of the axon initial segment. ASN Neuro 2017;9:1759091417745426.

82. Nimmerjahn A, Kirchhoff F, Helmchen F. Resting microglial cells are highly dynamic surveillants of brain parenchyma in vivo. Science 2005;308:1314-8.

83. Sierra A, Beccari S, Diaz-Aparicio I, Encinas JM, Comeau S, et al. Surveillance, phagocytosis, and inflammation: how never-resting microglia influence adult hippocampal neurogenesis. Neural Plast 2014;2014:610343.

84. Gordon S. Alternative activation of macrophages. Nat Rev Immunol 2003;3:23-35.

85. Martinez FO, Gordon S. The M1 and M2 paradigm of macrophage activation: time for reassessment. F1000Prime Rep 2014;6:13.

86. Ransohoff RM. A polarizing question: do M1 and M2 microglia exist? Nat Neurosci 2016;19:987-91.

87. Zhou T, Huang Z, Sun X, Zhu X, Zhou L, et al. Microglia polarization with M1/M2 phenotype changes in rd1 mouse model of retinal degeneration. Front Neuroanat 2017;11:77.

88. Peng H, Geil Nickell CR, Chen KY, McClain JA, Nixon K. Increased expression of M1 and M2 phenotypic markers in isolated microglia after four-day binge alcohol exposure in male rats. Alcohol 2017;62:29-40.

89. Chiu IM, Morimoto ET, Goodarzi H, Liao JT, O'Keeffe S, et al. A neurodegeneration-specific gene-expression signature of acutely isolated microglia from an amyotrophic lateral sclerosis mouse model. Cell Rep 2013;4:385-401.

90. Rosi S. A polarizing view on posttraumatic brain injury inflammatory response. Brain Circ 2016;2:126-8.

91. Keren-Shaul H, Spinrad A, Weiner A, Matcovitch-Natan O, Dvir-Szternfeld R, et al. A unique microglia type associated with restricting development of Alzheimer's disease. Cell 2017;169:1276-90.e17.

92. Friedman BA, Srinivasan K, Ayalon G, Meilandt WJ, Lin H, et al. Diverse brain myeloid expression profiles reveal distinct microglial activation states and aspects of Alzheimer's disease not evident in mouse models. Cell Rep 2018;22:832-47.

93. Leyns CEG, Ulrich JD, Finn MB, Stewart FR, Koscal LJ, et al. TREM2 deficiency attenuates neuroinflammation and protects against neurodegeneration in a mouse model of tauopathy. Proc Natl Acad Sci U S A 2017;114:11524-9.

94. Krasemann S, Madore C, Cialic R, Baufeld C, Calcagno N, et al. The TREM2-APOE pathway drives the transcriptional phenotype of dysfunctional microglia in neurodegenerative diseases. Immunity 2017;47:566-81.e9.

95. Mrdjen D, Pavlovic A, Hartmann FJ, Schreiner B, Utz SG, et al. High-dimensional single-cell mapping of central nervous system immune cells reveals distinct myeloid subsets in health, aging, and disease. Immunity 2018;48:599.

96. Deczkowska A, Amit I, Schwartz M. Microglial immune checkpoint mechanisms. Nat Neurosci 2018;21:779-86.

97. Poliani PL, Wang Y, Fontana E, Robinette ML, Yamanishi Y, et al. TREM2 sustains microglial expansion during aging and response to demyelination. J Clin Invest 2015;125:2161-70.

98. Wang Y, Cella M, Mallinson K, Ulrich JD, Young KL, et al. TREM2 lipid sensing sustains the microglial response in an Alzheimer's disease model. Cell 2015;160:1061-71.

99. Crotti A, Ransohoff RM. Microglial physiology and pathophysiology: insights from genome-wide transcriptional profiling. Immunity 2016;44:505-15. 
100. Chen SH, Oyarzabal EA, Hong JS. Critical role of the Mac1/NOX2 pathway in mediating reactive microgliosis-generated chronic neuroinflammation and progressive neurodegeneration. Curr Opin Pharmacol 2016;26:54-60.

101. Ransohoff RM, Khoury JE. Microglia in health and disease. Cold Spring Harb Perspect Biol 2016;8:a020560.

102. Kumar A, Barrett JP, Alvarez-Croda DM, Stoica BA, Faden AI, et al. NOX2 drives M1-like microglial/macrophage activation and neurodegeneration following experimental traumatic brain injury. Brain Behav Immun 2016;58:291-309.

103. Hool LC. Evidence for the regulation of L-type Ca2+ channels in the heart by reactive oxygen species: mechanism for mediating pathology. Clin Exp Pharmacol Physiol 2008;35:229-34.

104. Hool LC, Arthur PG. Decreasing cellular hydrogen peroxide with catalase mimics the effects of hypoxia on the sensitivity of the L-type Ca2+ channel to $\beta$-adrenergic receptor stimulation in cardiac myocytes. Circ Res 2002;91:601-9.

105. Hudasek K, Brown ST, Fearon IM. H2O2 regulates recombinant $\mathrm{Ca} 2+$ channel $\alpha 1 \mathrm{C}$ subunits but does not mediate their sensitivity to acute hypoxia. Biochem Biophys Res Commun 2004;318:135-41.

106. Mossakowski AA, Pohlan J, Bremer D, Lindquist R, Millward JM, et al. Tracking CNS and systemic sources of oxidative stress during the course of chronic neuroinflammation. Acta Neuropathol 2015;130:799-814.

107. Benned-Jensen T, Christensen RK, Denti F, Perrier JF, Rasmussen HB, et al. Live imaging of Kv7.2/7.3 cell surface dynamics at the axon initial segment: high steady-state stability and calpain-dependent excitotoxic downregulation revealed. J Neurosci 2016;36:2261-6.

108. Del Puerto A, Fronzaroli-Molinieres L, Perez-Alvarez MJ, Giraud P, Carlier E, et al. ATP-P2X7 receptor modulates axon initial segment composition and function in physiological conditions and brain injury. Cereb Cortex 2015;25:2282-94.

109. Evans MD, Sammons RP, Lebron S, Dumitrescu AS, Watkins TB, et al. Calcineurin signaling mediates activity-dependent relocation of the axon initial segment. J Neurosci 2013;33:6950-63.

110. Schafer DP, Jha S, Liu F, Akella T, McCullough LD, et al. Disruption of the axon initial segment cytoskeleton is a new mechanism for neuronal injury. J Neurosci 2009;29:13242-54.

111. von Bernhardi R, Heredia F, Salgado N, Muñoz P. Microglia function in the normal brain. Adv Exp Med Biol 2016;949:67-92.

112. Bilimoria PM, Stevens B. Microglia function during brain development: new insights from animal models. Brain Res 2015;1617:7-17.

113. Kato G, Inada H, Wake H, Akiyoshi R, Miyamoto A, et al. Microglial contact prevents excess depolarization and rescues neurons from excitotoxicity. eNeuro 2016;3:ENEURO.0004-16.2016.

114. Li Y, Du XF, Liu CS, Wen ZL, Du JL. Reciprocal regulation between resting microglial dynamics and neuronal activity in vivo. Dev Cell 2012;23:1189-202.

115. Wake H, Moorhouse AJ, Jinno S, Kohsaka S, Nabekura J. Resting microglia directly monitor the functional state of synapses in vivo and determine the fate of ischemic terminals. J Neurosci 2009;29:3974-80.

116. Tremblay MÈ, Lowery RL, Majewska AK. Microglial interactions with synapses are modulated by visual experience. PLoS Biol 2010;8:e1000527.

117. Beeton C, Garcia A, Chandy KG. Induction and clinical scoring of chronic-relapsing experimental autoimmune encephalomyelitis. J Vis Exp 2007:224.

118. Kipp M, Nyamoya S, Hochstrasser T, Amor S. Multiple sclerosis animal models: a clinical and histopathological perspective. Brain Pathol 2017;27:123-37.

119. Ransohoff RM. Animal models of multiple sclerosis: the good, the bad and the bottom line. Nat Neurosci 2012;15:1074-7.

120. Taetzsch T, Levesque S, McGraw C, Brookins S, Luqa R, et al. Redox regulation of NF-kB p50 and M1 polarization in microglia. Glia 2015;63:423-40.

121. Qin L, Wu X, Block ML, Liu Y, Breese GR, et al. Systemic LPS causes chronic neuroinflammation and progressive neurodegeneration. Glia 2007;55:453-62.

122. Denic A, Johnson AJ, Bieber AJ, Warrington AE, Rodriguez M, et al. The relevance of animal models in multiple sclerosis research. Pathophysiology 2011;18:21-9.

123. Dupree JL, Mason JL, Marcus JR, Stull M, Levinson R, et al. Oligodendrocytes assist in the maintenance of sodium channel clusters independent of the myelin sheath. Neuron Glia Biol 2004;1:179-92.

124. Torre-Fuentes L, Moreno-Jiménez L, Pytel V, Matías-Guiu JA, Gómez-Pinedo U, et al. Experimental models of demyelination and remyelination. Neurologia 2020;35:32-9.

125. Huang DW, Sherman BT, Lempicki RA. Bioinformatics enrichment tools: paths toward the comprehensive functional analysis of large gene lists. Nucleic Acids Res 2009;37:1-13.

126. Das A, Chai JC, Kim SH, Lee YS, Park KS, et al. Transcriptome sequencing of microglial cells stimulated with TLR3 and TLR4 ligands. BMC Genomics 2015;16:517.

127. Huang DW, Sherman BT, Lempicki RA. Systematic and integrative analysis of large gene lists using DAVID bioinformatics resources. Nat Protoc 2009;4:44-57.

128. Hanamsagar R, Alter MD, Block CS, Sullivan H, Bolton JL, et al. Generation of a microglial developmental index in mice and in humans reveals a sex difference in maturation and immune reactivity. Glia 2017;65:1504-20.

129. Hiremath MM, Saito Y, Knapp GW, Ting JP, Suzuki K, et al. Microglial/macrophage accumulation during cuprizone-induced demyelination in C57BL/6 mice. J Neuroimmunol 1998;92:38-49.

130. Fjær S, Bø L, Lundervold A, Myhr KM, Pavlin T, et al. Deep gray matter demyelination detected by magnetization transfer ratio in the cuprizone model. PLoS One 2013;8:e84162.

131. Dupree JL, Girault JA, Popko B. Axo-glial interactions regulate the localization of axonal paranodal proteins. J Cell Biol 1999; $147: 1145-52$.

132. Pomicter AD, Shroff SM, Fuss B, Sato-Bigbee C, Brophy PJ, et al. Novel forms of neurofascin 155 in the central nervous system: alterations in paranodal disruption models and multiple sclerosis. Brain 2010;133:389-405.

133. Geiss GK, Bumgarner RE, Birditt B, Dahl T, Dowidar N, et al. Direct multiplexed measurement of gene expression with color-coded 
probe pairs. Nat Biotechnol 2008;26:317-25.

134. Fruttiger M, Karlsson L, Hall AC, Abramsson A, Calver AR, et al. Defective oligodendrocyte development and severe hypomyelination in PDGF-A knockout mice. Development 1999;126:457-67.

135. Haure-Mirande JV, Audrain M, Fanutza T, Kim SH, Klein WL, et al. Deficiency of TYROBP, an adapter protein for TREM2 and CR3 receptors, is neuroprotective in a mouse model of early Alzheimer's pathology. Acta Neuropathol 2017;134:769-88.

136. Yamasaki R. Distinct roles of microglia and monocytes in central nervous system inflammation and degeneration. Clin Exp Neuroimmunol 2014;5:41-8.

137. Zrzavy T, Hametner S, Wimmer I, Butovsky O, Weiner HL, et al. Loss of 'homeostatic' microglia and patterns of their activation in active multiple sclerosis. Brain 2017;140:1900-13.

138. Li Q, Cheng Z, Zhou L, Darmanis S, Neff NF, et al. Developmental heterogeneity of microglia and brain myeloid cells revealed by deep single-cell rna sequencing. Neuron 2019;101:207-23.e10.

139. Hammond TR, Dufort C, Dissing-Olesen L, Giera S, Young A, et al. Single-cell RNA sequencing of microglia throughout the mouse lifespan and in the injured brain reveals complex cell-state changes. Immunity 2019;50:253-71.e6.

140. Barres BA, Hart IK, Coles HS, Burne JF, Voyvodic JT, et al. Cell death and control of cell survival in the oligodendrocyte lineage. Cell 1992;70:31-46.

141. Bennett ML, Bennett FC, Liddelow SA, Ajami B, Zamanian JL, et al. New tools for studying microglia in the mouse and human CNS. Proc Natl Acad Sci USA 2016;113:E1738-46.

142. Park MJ, Park HS, You MJ, Yoo J, Kim SH, et al. Dexamethasone induces a specific form of ramified dysfunctional microglia. Mol Neurobiol 2019;56:1421-36.

143. van Wageningen TA, Vlaar E, Kooij G, Jongenelen CAM, Geurts JJG, et al. Regulation of microglial TMEM119 and P2RY12 immunoreactivity in multiple sclerosis white and grey matter lesions is dependent on their inflammatory environment. Acta Neuropathol Commun 2019;7:206. 